\title{
RADIO EMISSION FROM EXTENDED SHELL-LIKE SNRS
}

\author{
A.I. ASVAROV \\ Institute of Physics, Azerbaijan Academy of Sciences, \\ Baku 370143,Azerbaijan Republic \\ e-mail: physic@lan.ab.az
}

\section{Introduction}

Observations of the soft X-Ray background and interstellar UV absorption lines have indicated that a large fraction of interstellar space is filled with a high temperature low density "coronal" gas. In such low density environments SNRs will expand up to $200 \mathrm{pc}$ in radius without thin shell formation which occurs due to radiative cooling effects. Such SNRs can occupy a large fraction of volume of Galaxy and can be the main source of background emissions. In the present work we examine the evolution of the radio emission of shell-like SNR evolving in the hot ISM.

\section{The model and results}

The main assumptions made in present study are the same as in our previous papers $[1,2]$ but now SNR is modeled by using an analytical approximation of [3] which follows the development of an adiabatic spherical blast wave in homogeneous ambient medium of finite pressure. At early times this approximation resembles the zero pressure Sedov similarity solution, but extends the range of investigation well into the regime in which the external pressure is significant.

At high Much numbers of the SNR's shock wave, $M_{s}$, emission highly concentrated directly behind the shock front but with decreasing of the shock strength the profile of distribution broadens. At $M_{s} \simeq 3$ the peak in profile of radial distribution separates from the shock front. SNRs at the end of their life (at $2 \leq M_{s} \leq 4$ ) have emissivity characteristics resemblint the mean characteristics of Galactic background synchrotron emission, namely, $\alpha \simeq 0.7$ and $\varepsilon_{1 G H z} \simeq 10^{-40}-10^{-41} \mathrm{erg} / \mathrm{cm}^{2} \mathrm{~s} \mathrm{sr} \mathrm{Hz}$. From the fact that the number of extended SNRs with small values of $M_{s}$ is considerable more then 
the number of young SNRs we can expect that such extended remnants occupy most of the galactic volume and are able to form galactic background synchrotron emission. The comparison of the modeled $\Sigma-D$ tracks with the observational $\Sigma-D$ relation for the shell-type SNRs (Fig.1) leads us to the conclusion similar to one made in [4], namely, adiabatic shell-type SNRs evolve at nearly constant $\Sigma$ followed by a steep decrease. In our model it begins when $M_{s} \leq 8-9$. The influence of the parameters of the ISM is more prominent then the parameters of $\mathrm{SN}$ explosion

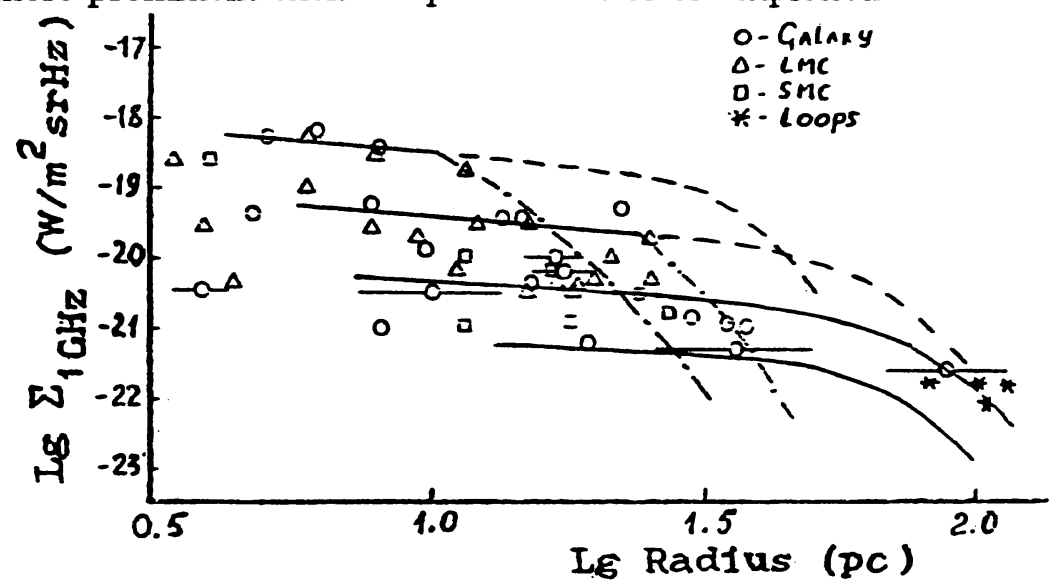

Fig.1. $\Sigma-R$ (Radius) diagram at $1 \mathrm{GHz}$ for shell-like SNRs. Data are taken from [5] and [6] for Galactic and MC SNRs, respectively. The positions of the giant loops are from [4]. Tracks are drawn (from bottom) for: (a) $n_{0 e}=10^{-3} \mathrm{~cm}^{-3}, P_{0}=5 \cdot 10^{3} \mathrm{~K}^{\prime} \mathrm{cm}^{-3}, H_{0}=3 \mu G$; (b) $n_{0 e}=5 \cdot 10^{-3} \mathrm{~cm}^{-3}$, $P_{0}=5 \cdot 10^{3} \mathrm{~K} \mathrm{~cm}^{-3}, H_{0}=5 \mu G$; (c) $n_{0 e}=0.5 \mathrm{~cm}^{-3}, P_{0}=5 \cdot 10^{3} \mathrm{~K} \mathrm{~cm}^{-3}, H_{0}=5$ $\mu G$; (d) $n_{0 e}=5 \mathrm{~cm}^{-3}, P_{0}=2 \cdot 10^{4} \mathrm{~K} \mathrm{~cm}^{-3}, H_{0}=10 \mu G$. The parts of tracks after beginning of radiative cooling are drawn by dashed and dot-dashed (no more acceleration) lines.

The main results are following: if the acceleration efficiency is such that one electron out $(2-3) 10^{3}$ thermal electrons is subjected to acceleration, then typical for the ISM magnetic fields compressed by a shock wave to a factor of four are sufficient for an explanation of the observed radio fluxes. The mean value of the spectral index of shell-like SNRs, evolving without setting in the radiative cooling, at the end of their life remains bounded at the value of $0.70-0.74$. The extended SNRs can serve as the main source of galactic nonthermal background radio emission.

References: [1] Asvarov A.I., 1992, AZh, v.69, p.753; [2] Asvarov A.I., 1994, AZh, v.71, p.228; [3] Cox D.P. and Anderson P.R., 1982,ApJ,v.253, p.268; [4] Berkhuijsen E.M., 1986,AA,166,257; [5] Green D.A., 1996, "A Catalogue of Galactic SNRs (August ver.)", MRAO, UK [6] Mathewson D.S. et al. 1983, ApJ (Suppl),51,345. 